\title{
La mujer "real": cuerpos e historias particulares en Real Women Have Curves de Josefina López
}

Anthony López Get

Escuela de Lenguas Modernas Universidad de Costa Rica

\begin{abstract}
Resumen
Este artículo analiza las relaciones de poder existentes entre los sujetos "hombres" y "mujeres" en la obra de Josefina López Real Women Have Curves. Desde un enfoque feminista poscolonial, se estudia la dominación masculina sobre el cuerpo de las mujeres - cuerpos colonizados -, así como la necesidad, planteada en la obra, de un estudio feminista multicultural - no etnocéntrico-, incluyente, que tome en cuenta las particularidades de las mujeres doblemente marginadas en tanto mujeres del Tercer Mundo en oposición a una identidad de mujer absoluta y esencialista.
\end{abstract}

Palabras claves: feminismo, poscolonialismo, inmigrantes, identidad, género

\begin{abstract}
This article analyzes the power relations between the subjects "men" and "women" in Josefina López' play Real Women Have Curves. From a postcolonial feminist approach, we study the male domination over the female body - as colonized bodies - and the need, as suggested in the play, of a multicultural - not ethnocentric-, inclusive, feminist study that takes into consideration the particularities of the double-marginalized women (as women and as women from developing countries) as opposed to an absolute and essentialist identity.
\end{abstract}

Key words: feminism, postcolonialism, immigrants, identity, gender

$\mathrm{E}$ $n$ la obra teatral Real Women Have Curves, la autora, Josefina López, explora la vida de un grupo de mujeres inmigran- tes que laboran en la industria textil en los Estados Unidos. Con un cambiante tono que va del humor a la tristeza, López abarca las distintas posiciones y 
puntos de vista que las protagonistas de esta obra tienen del ser "mujer" y "chicana", en un contexto de discriminación, persecución e ilegalidad. Los personajes se ubican en posiciones particulares que se determinan por la edad, la posición económica, el nivel de educación, la maternidad o su imposibilidad, la sexualidad y la apariencia física, todos aspectos que influyen en la visión de mundo de todas y cada una de estas mujeres y que las llevan incluso a enfrentarse unas contra otras por choques de ideas y de percepciones.

Ana, personaje principal cuya educación la pone en una aparente posición de ventaja con respecto a los demás personajes, ve cómo su conocimiento y su conciencia feminista - que ve a la mujer como un sujeto determinado - ceden ante la riqueza que cada mujer aporta a su mundo desde posiciones muy particulares, no esenciales. Son temas centrales de esta obra la posición subordinada de la mujer en una sociedad patriarcal, aunado a unas condiciones económicas que las mantienen sujetas, limitadas, en su desarrollo personal. No obstante, la autora no solo denuncia la dominación patriarcal sobre "la mujer" y la dominación económica sobre el inmigrante en los Estados Unidos, sino que critica, de forma sutil, el etnocentrismo de un movimiento feminista que deja de lado las particularidades de algunas "mujeres" que no encajan en un modelo universalista de Primer Mundo, y plantea no solo la necesidad de los estudios feministas de tomar en cuenta la etnia, la cultura, la clase social y la religión, como factores determinantes de la realidad de los distintos grupos de mujeres para entablar una lucha efectiva e incluyente, sino también explora las formas alternativas de pensamiento $\mathrm{y}$ de concepción del "ser" mujer como lugares de resistencia.

La lucha feminista tradicional se ha enfocado en poner en evidencia la dominación masculina en diferentes sociedades y la posición subordinada en la que las mujeres han sido colocadas. Al revelar la dominación masculina como un constructo ideológico que se ha "naturalizado" en todos los aspectos de las relaciones humanas, el feminismo busca revertir, en algunos casos, o anular las diferencias creadas en aras de una relación equitativa de género. Bourdieu comenta sobre las estructuras de dominación masculina, las cuales son "el producto de un trabajo continuado (histórico por tanto) de reproducción al que contribuyen unos agentes singulares (entre los que están los hombres, con unas armas como la violencia física y la violencia simbólica) y unas instituciones: Familia, Iglesia, Escuela, Estado" (50). El autor evidencia cómo las estructuras de poder actúan en la reproducción de la dominación masculina y la subyugación femenina, no solo con la violencia física, sino también simbólicamente, por medio, y haciendo eco de Althusser, de los Aparatos Ideológicos Estatales o, para ser más específicos, aparatos ideológicos patriarcales. Esta dominación simbólica, inmersa en la trama institucional de una sociedad, tiene como resultado lo que Millet llama interior colonization (colonización interna), que lleva tanto a mujeres como a hombres a creer en el derecho natural de los últimos a dominar a las primeras, dominación que se toma como el orden natural de las cosas, como un derecho adquirido de nacimiento por los hombres, y que supera a otros tipos de 
dominación ideológica por su sutileza y arraigo (25). Tal y como lo expone Millet, "Male supremacy, like other political creeds, does not finally reside in physical strength but in the acceptance of a value system which is not biological" (27). Es la aceptación de una "realidad" inventada, de una superioridad construida a través de la historia y que ha venido a institucionalizarse y a naturalizarse en nuestro quehacer diario como si fuese el obvio resultado de una serie de características innatas en los seres humanos. Desde esta perspectiva, Cixous lo explica como

Subordinación de lo femenino al orden masculino que aparece como la condición del funcionamiento de la máquina. La puesta en duda de esta solidaridad entre el logocentrismo y el falocentrismo se ha convertido, hoy en día, en algo urgente ... para amenazar la estabilidad del edificio masculino que se hacia pasar por eterno-natural; haciendo surgir, en lo que se refiere a la feminidad, reflexiones, hipótesis necesariamente ruinosas para el bastión que aún detenta la autoridad. (16)

El feminismo, por tanto, busca minar las bases ideológicas de la dominación masculina para así darle a las mujeres la posibilidad de crecer más allá de los parámetros establecidos por una sociedad patriarcal, y de liberarse de su posición subalterna. No obstante, esta búsqueda de una identidad femenina se ha caracterizado por buscar "una" identidad que abarque a todas las mujeres del mundo independientemente de otras condiciones de existencia que, al cabo, imposibilitan o limitan la coordinación efectiva de prácticas y pensamientos de resistencia.
Femenías explica, “«Identidad» es un concepto estabilizador donde sólo hay cambio; genera un dispositivo normalizador, homologador que subsume las diferencias y crea la fantasía de la unidad y de la pureza" (186). Si bien la ideología patriarcal impone "identidades" definidas a hombres y mujeres (en sus categorías más básicas) para "normalizar" las relaciones de dominación masculina, de igual forma el feminismo había caído en el mismo juego, al tratar de establecer una identidad femenina homóloga a todas las mujeres del mundo, para también "normalizar" la lucha en fines comunes muy definidos. No obstante, la realidad local en la que las mujeres se desarrollan ha demostrado que dicha homología es, en palabras de Femenías, una "fantasía", en tanto existe una gran variedad de factores que particularizan la situación de cada mujer, y por supuesto de cada hombre, y que vuelve la lucha "normativa" mucho menos efectiva. En este sentido, Butler hace un llamado a reexaminar el enfoque y la metodología feministas en tanto esencialistas. Nos dice, “... insistir en la coherencia y la unidad de la categoría de las mujeres ha negado, efectivamente, la multiplicidad de intersecciones culturales, sociales y políticas en que se construye el conjunto concreto de "mujeres" (47). Butler añade elementos culturales, sociales y políticos como variables determinantes en la construcción de las distintas concepciones de mujer en diferentes contextos, y añade que "La crítica feminista debe explorar las afirmaciones totalizadoras de una economía significante masculinista, pero también debe ser autocrítica respecto de los gestos totalizadores del feminismo" (46). La lucha feminista se torna "totalizadora" 
al obviar las particularidades de cada individuo, en lo que Castillo llama un "etnocentrismo del feminismo hegemónico" (207), y al crear al sujeto femenino basándose en un modelo occidental de primer mundo.

En la misma línea, Femenías comenta, "Incluso el feminismo tal como Occidente lo ha definido apropiándoselo es sólo una forma de imperialismo cultural" (160). La lucha feminista, que si bien es una lucha antihegemónica, se torna excluyente y hasta discriminatoria al pasar por alto las realidades de los sujetos más allá de la realidad occidental. "El cumplimiento de promesas de la modernidad [incluyendo la promesa feminista] es posible sólo para aquellos que hablan su lenguaje", comenta Belausteguigoitia (230), poniendo así de manifiesto la desigualdad en el acceso a los procesos liberadores, de cambio social para aquellas que se ubican en una marginalidad que va más allá del simple hecho de ser mujer (una marginalidad doble, triple, etc.), que no poseen los medios para "hablar" el mismo idioma de los grupos de resistencia hegemónicos, tan contradictorio como pueda parecer. Con esto, según Bourdieu “... corremos el peligro, por tanto, de recurrir, para concebir la dominación masculina, a unos modos de pensamiento que ya son el producto de la dominación" (17). Utilizar el "lenguaje" y los mismos medios de discriminación culturalmente hegemónica para revertir las relaciones de género, anula la efectividad de esta pretendida lucha universal por el bien de todas las mujeres. Castillo da un ejemplo de cómo el "feminismo hegemónico" afecta la igualdad dentro del mismo movimiento. Nos dice: "Es importante reconocer que las desigualdades étnicas y de clase influyen en que, aunque sea de manera no intencionada, las mujeres mestizas [en contraste con las mujeres indígenas], con un mejor manejo del español y de la lecto-escritura, tendamos a hegemonizar la discusión cuando se trata de espacios conjuntos" (224). En este caso específico, de la experiencia personal de Castillo, el nivel de educación formal se convierte en un agente de división de poder dentro del mismo movimiento de lucha lucha que pone a las activistas "menos educadas" en la lengua hegemónica en una posición de desventaja.

Por otro lado, Butler analiza la problemática de la identidad de género argumentando que no existe un sujeto esencial previo a la construcción identitaria. Comenta que:

... el género resulta ser performativo, es decir, que constituye la identidad que se supone que es. En este sentido, el género es un hacer, aunque no un hacer por parte de un sujeto que se pueda considerar preexistente a la acción. El desafío que implica replantear las categorías de género fuera de la metafísica de la sustancia tendrá que tomar en cuenta la pertinencia de la afirmación que hace Nietzsche en $L a$ genealogía de la moral en cuanto a que "no hay ningún "ser" detrás del hacer, del actuar, del devenir; "el agente" ha sido ficticiamente añadido al hacer, el hacer es todo' no hay una identidad de género detrás de las expresiones de género; esa identidad se constituye performativamente por las mismas "expresiones" que, según se dice, son resultado de ésta. (58)

La performatividad crea la identidad, crea al sujeto. Son las "expresiones" 
creadas sobre el género lo que define a los sujetos mujer y hombre. No hay un sujeto esencial, absoluto, primordial, al que podamos atribuirle la característica de "ser mujer", ya que este depende de las construcciones ideológicas en las que se desarrolla. Por ende, un discurso feminista emancipatorio que reúna a todos y cada uno de los sujetos "mujeres" como un absoluto universal estaría generando una nueva identidad creada, impuesta, discriminatoria, unidimensional, que obvia las condiciones de existencia de sujetos alternativos al sujeto occidental.

La crítica feminista, por tanto, debe atender a las distintas fuerzas que influyen en la construcción de los sujetos particulares en condiciones de existencia también particulares. Butler propone que:

Si una es una mujer, desde luego eso no es todo lo que una es; el concepto no es exhaustivo, no porque una "persona" con un género predeterminado trascienda los atributos específicos de su género, sino porque el género no siempre se establece de manera coherente o consistente en contextos históricos distintos, y porque se interseca con modalidades raciales, de clase, étnicas, sexuales $y$ regionales de identidades discursivamente constituidas. (35, la cursiva es mía)

Butler introduce una historicidad en la construcción identitaria de los sujetos que no solo se definen como "mujeres" sino que son también atravesadas por la raza, la etnia, y muchas otras variantes. De forma similar, Castillo critica el discurso feminista cuando este pierde de vista las particularidades de un grupo de mujeres específico -en este caso, las mujeres indígenas-. Nos dice que "los discursos generalizadores del feminismo que enfatizan el derecho a la igualdad, sin considerar la manera en que la clase y la etnicidad marcan las identidades de las mujeres indígenas" (218). No es posible hablar de una igualdad en general, en tanto que las condiciones de existencia específicas, atravesadas por la cultura, la religión, la etnia, entre muchos otros factores, influyen en las necesidades particulares de mujeres en contextos distintos de los de las mujeres occidentales ${ }^{1}$, por poner un ejemplo. Esta perspectiva sobre la nueva tarea del feminismo es de suma importancia, en tanto el cambio efectivo hacia una equidad entre géneros dependerá de la conciencia no solo de las similitudes entre los sujetos, sino también de las diferencias, pues lo que es bueno para unas, quizá no lo sea tanto o tan fácil de asimilar para otras.

Tal y como lo expone Bourdieu, "Las pasiones del hábito dominante (desde la perspectiva del sexo, de la etnia, de la cultura o de la lengua), relación social somatizada, ley social convertida en ley incorporada, no son de las que cabe anular con un mero esfuerzo de la voluntad, basado en una toma de conciencia liberadora" (55). Si pensamos en la dificultad que representa para las sociedades revertir las condiciones establecidas sobre género, este proceso se puede tornar inmanejable en contextos donde la cultura, por ejemplo, choca con algunas premisas del discurso feminista. Castillo ve esta problemática como “... un llamado de atención al movimiento feminista urbano sobre la necesidad de construir un feminismo multicultural que reconozca las distintas maneras en que las mujeres mexicanas [como un ejemplo 
particular] imaginan sus identidades de género o conciben sus estrategias de lucha" (207). Al tomar en cuenta las distintas construcciones identitarias definidas no solo por el género, sino por múltiples variables sociales, culturales, económicas, el feminismo deja de ser excluyente, etnocéntrico, puede encontrar soluciones adecuadas al contexto de cada grupo y le da una voz a quienes eran silenciadas por sus propias compañeras de lucha.

Para analizar las relaciones de género en un contexto latinoamericano por ejemplo -como es el caso de la obra de López que estudiaremos más adelante- se deben tomar en cuenta las relaciones de género en un contexto poscolonial aunado a un mundo globalizado con políticas económicas que afectan la región de manera distinta que a otras regiones tanto del Primer como del Tercer Mundo. Estos factores acentúan las diferencias de clase, de etnia, de educación, entre otros, tanto mundiales como locales. Tal y como lo expresa Mohanty, “... el trabajo feminista transcultural debía poner atención a las micropolíticas de contexto, subjetividad y lucha, así como a la macropolítica de los sistemas y procesos políticos y económicos globales" (411). No basta con centrarse en las particularidades de un grupo específico, sino que se deben tomar en cuenta también las interrelaciones producto de las tendencias globalizantes y de cómo todo el conjunto de factores (micro y macro) afectan la percepción de dicho grupo. Femenías comenta cómo, en el contexto actual, los procesos de globalización en conjunto con las creencias y valores locales han potenciado las formas de exclusión hasta niveles impensables (182). Mohanty explica también los efectos de las políticas económicas globales impuestas por el Primer Mundo sobre los sujetos del Tercer Mundo; nos dice: "Los efectos de estos cuerpos gobernantes sobre la gente pobre del mundo han sido devastadores. En formas importantes, han sido las mujeres y niñas del mundo, especialmente del Tercer Mundo/Sur, quienes llevan la carga más pesada de la globalización" (430). Este fenómeno al que se refiere Mohanty es de vital importancia para reconocer las posibles implicaciones que pueda tener en las relaciones de género de un país o región del Tercer Mundo en comparación con uno del Primer Mundo.

Un enfoque poscolonial ${ }^{2}$ toma en consideración los factores tanto regionales como globales, las condiciones de dependencia que el grupo "ex"colonizado tiene con los agentes "ex"colonizadores, y los efectos políticos, sociales, culturales y demás, que esta relación puede generar en los sujetos tanto a lo interno del grupo como en el nivel global. Al respecto Young comenta: "Postcolonial feminism is certainly concerned to analyse the nervous conditions of being a woman in a postcolonial environment, whether in the social oppression of the postcolony or the metropolis" (115); y luego agrega: "The specific conditions, therefore, for women in postcolonial states, or the postcolonial conditions in metropolitan states for migrants, vary according to location, with the result that there cannot be a single form of postcolonial politics" (113). Los estudios poscoloniales amplían su rango a las relaciones del sujeto poscolonial en diferentes instancias dependiendo de donde se ubica incluso geográficamante: en el centro o en la periferia. Al decir que no 
existe una única forma de política postcolonial, Young alude a los múltiples factores que se deben tomar en cuenta para definir las necesidades que un grupo de mujeres específico tiene. Por ejemplo, no podemos plantear de igual manera las necesidades de una costarricense mestiza, de clase media, con educación universitaria, a una indígena costarricense pobre, sin educación formal, o a una costarricense caucásica inmigrante ilegal en los Estados Unidos, o a una costarricense negra de clase alta que estudia en Yale, mucho menos a una mujer francesa de clase media, estudiante en La Sorbona. Si bien todas son mujeres, todas y cada una de ellas vienen de mundos distintos, de realidades totalmente ajenas a las otras, con experiencias de vida que forjan la concepción de lo que para ellas es "ser" mujer y que crean distintas necesidades en cuanto a la lucha de géneros se refiere.

No obstante, el feminismo tradicional, como lo mencionamos anteriormente, habría favorecido a la mujer francesa como representante universal de "la mujer". Femenías explica que “...el feminismo postcolonial pretende subvertir la desvalorización histórica no sólo de las mujeres, sino también de la etnia (cultura, nación) a la que pertenecen, inferiorizada por la colonización, y resignificar positivamente las diferencias entendidas como recíprocas" (160) y propone, sobre este tipo de enfoque, que "El análisis postcolonial del atravesamiento del sujeto por variables como el sexo, la etnia, la clase, la religión, la opción sexual u otras, inevitablemente socava las fronteras identitarias reguladas por las narrativas dominantes" (159). En otras palabras, el enfoque poscolonial le arreba- ta el discurso de resistencia feminista a grupos que perpetuán otras formas de discriminación y subyugación, que a fin de cuentas atentan contra el propio fin del feminismo como discurso de rebelión. La obra Real Women Have Curves ilustra perfectamente la problemática que el feminismo enfrenta, al presentar a cinco mujeres que, si bien se enfrentan a situaciones similares a las que cualquier mujer en una sociedad patriarcal se enfrenta y que además provienen de contextos muy similares que las unen - como el hecho de ser inmigrantes -, se ven envueltas en choques ideológicos provocados por variables específicas que determinan lo que cada una considera "ser mujer". Si bien la obra explora temas que el feminismo tradicional ha enfatizado - tales como el cuerpo femenino y la sexualidad, la maternidad, los derechos laborales, la violencia - lo hace desde una perspectiva muy particular, mostrando cómo Ana, la voz del feminismo en la obra, finalmente aprende que su visión de mundo no necesariamente se adapta a la realidad de todas las mujeres en todos los contextos.

La obra de López presenta a cinco mujeres latinas que viven y trabajan en los Estados Unidos, recientemente legales, con excepción de una, todas con algún nivel de sobrepeso -tema recurrente en la obra-, y que laboran en una fábrica de ropa que pertenece a una de ellas. Estudiemos brevemente las características de cada una. Ana es la protagonista de la obra; es la menor de las cinco (18 años) y tiene un nivel de educación formal más alto que las demás -es de la única que se menciona haber realizado algún tipo de estudio, además de su hábito de lectura- que la ha puesto en contacto con una visión de 
mundo distinta de la del resto de su familia. Es una feminista declarada que aspira a ir a la universidad para convertirse en escritora y parece ser la que se ha adaptado mejor a la nueva cultura en la que viven. Su hermana Estela, de 24 años, es la dueña de la fábrica donde se desarrolla la obra y el único personaje que continúa siendo ilegal. Si bien no posee un nivel de educación relevante y no es una activa partidaria del movimiento feminista, quizá por una cuestión meramente generacional, sí se proyecta como una mujer decidida a ser más que una empleada, una madre o incluso un objeto sexual. Carmen de 48 años y madre de Ana y Estela (y de muchos hijos más), y Pancha de 32 años, convergen en un conflicto por la maternidad. La primera sufre al creer estar embarazada una vez más, mientras que la otra sufre al no poder ser madre. Finalmente, Rosalí de 29 años se presenta como una mujer profundamente preocupada por su apariencia física, cuya meta en la vida - al menos en un futuro inmediato - es llegar a ser talla siete. Todas estas particularidades las llevan a discutir temas sobre "la mujer" desde perspectivas y posiciones distintas.

Como representante del feminismo, durante una discusión con las demás, Ana expone sus ideas sobre los derechos de "las mujeres" y la posición pasiva de sus compañeras: "I don't know everything, but I know a lot. I read a lot. But it just amazes me to hear you talk the way you do. A women's liberation movement happened 20 years ago, and you act like it hasn't happened" (1.3.34). Desde su posición de mujer "más educada", con una fuerte conciencia de género, Ana se adjudica el derecho de juzgar al resto de mujeres que aún siguen bajo el yugo de la ideología patriarcal por no hacer nada para liberarse, aun cuando las condiciones para hacerlo, desde su punto de vista, están dadas. No obstante, Pancha le abre los ojos a Ana sobre la realidad de otras mujeres que no se sienten identificadas con el movimiento de liberación femenina hegemónico: "Mira, all those gringas shouting about liberation hasn't done a thing for me..." (1.3.34). Desde su posición de inmigrante latina, sin educación, trabajando por el salario mínimo en condiciones inhumanas, la liberación femenina no tiene para Pancha gran sentido cuando la supervivencia en un mundo hostil es su prioridad. ¿En qué ha ayudado el feminismo a Pancha para solucionar no solo su situación como mujer oprimida, sino su situación como mujer inmigrante oprimida? Esta disyuntiva entre la posición de Ana como feminista y la posición de las demás mujeres como muchas otras cosas además de mujeres, permea las discusiones relativas a la identidad de la mujer. Finalmente Ana reconoce las limitantes del pensamiento feminista absoluto al reconocer en las posiciones alternativas focos de resistencia,

I always took their work for granted, to be simple and unimportant. I was not proud to be working there at the beginning. I was only glad to know that because I was educated, I wasn't going to end up like them. I was going to be better than them. And I wanted to show them how much smarter and liberated I was. I was going to teach them about the women's liberation movement, about sexual liberation and all the things a so-called educated American woman knows. But in their subtle ways they taught me about resistance. 
About a battle no one was fighting for them except themselves. About the loneliness of being women in a country that looks down on us for being mothers and submissive wives. With their work that seems simple and unimportant, they are fighting ... Perhaps the greatest thing I learned from them is that women are powerful, especially when working together... (2.4.69)

Ana descubre que lo que el Primer Mundo le ofrece a ella no necesariamente se adapta a las realidades de sus compañeras. Ese tono de superioridad con el que Ana solía pensar acerca de las otras mujeres -verlas como pobres, simples y tontas mujeres destinadas a la maquila de por vida- representa, por un lado, la visión del Primer Mundo para con los inmigrantes del Tercer Mundo, y por el otro, la visión del feminismo del Primer Mundo para con las mujeres que "no quieren adaptarse" al cambio. No obstante, su cambio de mentalidad representa el necesario movimiento del feminismo hacia una visión incluyente de las distintas condiciones de existencia y de los distintos contextos que determinan a las mujeres. Este cambio de perspectiva en Ana se da a través de un proceso de reconocimiento de la forma en que estas mujeres viven aspectos tales como la sexualidad y el cuerpo, la maternidad, la violencia, aspectos comunes en la crítica al patriarcado, pero desde posiciones doblemente marginadas como mujeres y como inmigrantes latinoamericanas.

La clase social representa uno de los aspectos más relevantes en la construcción de identidades femeninas poscoloniales, en tanto está directamente ligada al acceso a la educación, a la subordinación en el trabajo y a la acep- tación de la subordinación de género en un marco de discriminación por etnia y por estatus migratorio. Tal y como lo expone Mohanty,

Así como las fábricas y los lugares de trabajo de las corporaciones globales buscan y disciplinan el trabajo de mujeres pobres del Tercer Mundo/ Sur,inmigrantes/emigrantes, de la misma manera las cárceles de Europa y de los Estados Unidos aprisionan a números desproporcionadamente altos de mujeres de color, inmigrantes y no ciudadanas, de ascendencia africana, asiática y latinoamericana. (451)

El inmigrante, ya sea legal o ilegal, siempre es visto como ajeno, como extraño, como fuera de la ley, y su posición ante los ojos de las sociedades del Primer Mundo es la de un subordinado apto para el trabajo duro y mal pagado. Esta condición afecta particularmente a las mujeres, quienes llevan la doble carga de ser inmigrantes y mujeres una doble subordinación-. La obra de López refleja las consecuencias que esta doble marginalización tiene sobre las trabajadoras inmigrantes y sus aspiraciones. En primera instancia, la obra constantemente expone situaciones en las que las mujeres temen ser deportadas, aun cuando el estatus migratorio de la mayoría es legal,

CARMEN. ¡La migra! (All the WOMEN scatter and hide waiting to be discovered. Then after a few seconds PANCHA makes a realization).

PANCHA. Pero, why are we hiding? We're all legal now.

CARMEN. ¡Ayy, de veras! I forget! All those years of being an ilegal, I still can't get used to it. (...) 
ANA. Thank God, I'm legal. I will never have to lie on applications anymore, except maybe about my weight... (1.1.14-15)

El olvido de su condición de inmigrante legal es un síntoma de la marginalización sistemática que sufren los inmigrantes, donde su marginalidad es constantemente recordada. El hecho expuesto por Ana de que la palabra en inglés alien se refiere tanto a los inmigrantes como a los extraterrestres (1.1.10) refleja un subtexto en el discurso hegemónico, donde el "alien" siempre será, independientemente de su estatus migratorio, un agente externo, extraño, extra. Por otra parte, Ana, a pesar de ser, como lo mencionamos anteriormente, la que más se ha adaptado a la cultura estadounidense, ve con frustración su situación actual, al no tener los recursos para seguir adelante con sus estudios y al verse obligada a trabajar bajo malas condiciones,

... Is it selfish of me not to want to wake up every morning at 6:30 a.m., Saturdays included, to come here for 67 dollars a week? Oh, but such is the life of a Chicana in the garment industry. Cheap labor... I've been trying to hint my sister for a raise, but she says I don't work fast enough for her to pay me minimum wage. The weeks get longer and I can't believe I've ended up here. I just graduated from high school... Most of my friends are in college... It's as if I'm going backwards. ... What I really want to do is write... (1.1.10, la cursiva es mía)

Ana reconoce su situación como lo típico para una chicana. No solo el trabajo es duro y mal pagado, sino que además la sujeta a una condición económica de mera subsistencia, de la que nunca obtendrá los frutos necesarios para costear su desarrollo profesional a no ser que consiga ayuda económica del propio Estado que la condiciona a su posición actual. Más adelante, Ana compara su posición con la de una antigua amiga,

It feels just as bad as when I was doing the fries at McDonald's. Pouring frozen sticks of potatoes into boiling lard and the steam hitting my face for $\$ 3.35$ an hour... This place stinks! I hate going to the store and having to climb over the winos, and ignore the catcalls of the sexist dope addicts and the smell of urine and marijuana on the street, and... I went to the store today and I saw an old friend. She's pregnant, again. She says she's happy and she doesn't care if she's on welfare. When she was still in high school she told me she knew I was going todo something with my life. I don't want her to know I work here. (1.5.40)

Podemos ver dos de las opciones más claras que una mujer inmigrante tiene como forma de vida: como trabajadora explotada y como madre. Para Ana, ninguna de las opciones es atractiva; sin embargo, se siente atrapada por su condición socioeconómica y se avergüenza de ello. Para Ana, la posición de su amiga -conformarse con una vida de mujer constantemente embarazada que sobrevive con la ayuda económica del Estado- es algo inconcebible, en tanto reconoce en sí misma la capacidad intelectual para llegar mucho más lejos; no obstante, ella no logra reconocer la posibilidad de que su amiga sea realmente feliz (el texto no nos revela esta información) con su 
vida de madre, ya que dicha condición no concuerda con su idea de "hacer algo con su vida".

La obra explora también las condiciones de explotación que las mujeres trabajadoras sufren en la industria textil. No sólo los salarios bajos, sino las condiciones propias de la actividad, son formas violentas de sometimiento que toman ventaja de su situación como mujeres y como inmigrantes. Carmen comenta, "Pos yo ya no veo. I can't see a thing. ... I should retire and be an abuelita by now, taking care of grandchildren... I don't know why I work, I have arthritis in my hands, I'm losing my sight from all the sewing, and this arm, I can hardly move it anymore..." (1.1.11). El sometimiento a condiciones inhumanas de trabajo afecta a estas mujeres física y psicológicamente, no solo por ver su salud deteriorada sino también sus sueños frustrados. Los ejemplos de explotación son varios, desde los plazos que se les dan a estas mujeres para cumplir con las entregas, a lo que Pancha se refiere como un trato de "máquinas" y no de humanos (1.1.18), hasta el valor que se le da al producto de su trabajo. Comentan,

ANA. How much do they pay us for making these dresses?

ROSALI. Estela, we get thirteen dollars for these, no?

ANA. Oh, yeah? How much do they sell them for at the stores?

ESTELA. They tell me they sell them at Bloomingdale's for about two hundred dollars.

WOMEN. iiQuée!! (1.1.22)

La diferencia en el valor de un vestido colgado en la "Fábrica García" al mismo vestido en una vitrina en
Bloomingdale's es absurdo y denota el nivel de explotación a que estas mujeres han sido relegadas. Ana reflexiona sobre las diferencias de estatus entre las mujeres inmigrantes y las mujeres del Primer Mundo,

(to PANCHA). It's not that I don't iron fast enough, it's that whenever I finish ironing a dress I stop for a minute to really look at it. I never realized just how much work, puro lomo, as my mother would say, went into making it. Then I imagine the dress at Bloomingdale's and I see a tall and skinny woman looking at it. She instantly gets it and with no second thoughts she says "charge it!" She doesn't think of the life of the dress before the rack, of the labor put into it. I shake the dress a little and try to forget it's not for me. I place a plastic bag over it then I put it on the rack and push it away. It happens to me with every dress. (2.1.50)

Ana reconoce con pesar que entre ellas y la mujer que compra en Bloomingdale's existe una enorme diferencia que las separa. Sus realidades, sus condiciones de existencia son totalmente opuestas. Unas fabrican un vestido con mucho trabajo para obtener $\$ 13$ para sobrevivir, mientras que las otras no se preocupan en gastar más de $\$ 200$ por él, ya que su supervivencia no depende de ese dinero. Estas dos posiciones, por supuesto, cambian la forma en que todas estas mujeres perciben el mundo y "la realidad" del "ser mujer".

Otro aspecto que desarrolla la obra de López es la corporalidad como determinante del "ser mujer". El cuerpo femenino es objeto de valor, en el sentido en que se torna un objeto en tanto posesión y que tiene un valor 
tanto mercantil -como trabajadoras explotadas- como simbólico de lo que una mujer completa debe ser. La dominación del cuerpo de la mujer está presente en la mayoría de las culturas en aspectos tales como la violencia, la libertad sexual -o la falta de ella-, la apariencia física en función de los deseos del hombre y la maternidad como la realización de la mujer, entre otros. Millet comenta, "Traditionally, patriarchy granted the father nearly total ownership over wife or wives and children, including the powers of physical abuse and often even those of murder and sale" (33). Si bien es cierto que gracias a los logros del feminismo este control sobre la vida de las mujeres no es tan evidente, tan violento, ni tan frecuente como en el pasado -al menos en la mayoría de las sociedades de Occidente donde es penalizado-, los rezagos de esta dominación siguen latentes en diferentes formas de control violentas tanto físicas como psicológicas. El cuerpo de la mujer, en especial en contextos con una tradición patriarcal fuertemente arraigada en la cultura -como es el caso latinoamericano-, sigue estando fuera del control de la propia mujer. Cixous nos dice que la mujer "No ha podido habitar su "propia» casa, su propio cuerpo" (20) y comenta como "No han tenido ojos para ellas mismas. No han ido a explorar su casa. Su sexo les asusta aún ahora. Les han colonizado el cuerpo del que no se atreven a gozar. La mujer tiene miedo y asco de la mujer" (21). Las mujeres habitan un territorio colonizado, que no les pertenece en realidad, y sobre el cual no tienen control. Esta forma de dominación sobre el cuerpo de la mujer se hace evidente en aspectos tales como la apariencia física y la sexuali- dad. Por un lado, explica Cixous, "[las mujeres] Nos hemos apartado de nuestros cuerpos, que vergonzosamente nos han enseñado a ignorar, a azotarlo con el monstruo llamado pudor" (58). En la misma línea, Bourdieu nos habla sobre la moral del cuerpo,

... sentimientos vinculados a las diferentes partes del cuerpo, a la espalda que hay que mantener erguida, a los vientres que hay que disimular, a las piernas que no deben estar abiertas, etc., posturas todas ellas que están cargadas de una significación moral ... Como si la feminidad se resumiera en el arte de «empequeñecerse» (43).

La idea de "empequeñecerse" que nos presenta Bourdieu implica no sólo ocultar el ser sexual que habita en cada mujer, sino también minimizar los elementos del cuerpo que no van acordes con el ideal de belleza al ocultar una panza prominente, unos glúteos caídos, o la celulitis, por poner algunos ejemplos.

Los personajes de López se enfrentan a una doble colonización: la colonización territorial que si bien políticamente ya no existe, ideológica y económicamente continúa con gran fuerza, y a la colonización del cuerpo que menciona Cixous. En primera instancia, encontramos la violencia física como derecho de los hombres sobre el cuerpo y la mente de las mujeres. Si bien podemos encontrar casos de violencia hacia las mujeres en todo el mundo, es en los países del Tercer Mundo donde a causa de una tradición patriarcal fuertemente arraigada a la cultura, aunado al problema de la pobreza y los bajos niveles de educación, los casos de agresión -llámese golpiza, 
maltrato verbal, violación, etc.- se integran y se asimilan a la vida diaria como algo normal. Tal es el caso en la obra cuando las mujeres escuchan un programa de radio sobre el tema:

ESPERANZA (voice-over). For those of you who just joined us today we are discussing abusive spouses. We have our last caller on the line. Caller, are you there?

CALLER (voice-over). Hi. I'm not going to give you my name because my husband listens to this station. I wanted to know what I can do to... Well, I want to know how I can talk to my husband when he gets angry.

ESPERANZA (voice-over). How long has he been abusive?

CALLER (voice-over). Ah... Well, he wasn't like this when we got married... He was always sweet. So I don't know what has happened to him. He tells me if I did whatever he asked he wouldn't have to hit me. But I do what he says and it's still not good enough. Last time he hit me because... [...]

CARMEN. Pobre mujer, I'm lucky mi viejo doesn't hit me.

ANA. Lucky? Why lucky? It should be expected that he doesn't. That woman should leave her husband. Women have the right to say "no."

PANCHA. You think it's that easy?

ANA. No, she's probably dependent on him financially, or the church tells her to endure, or she's doing it for the children.

PANCHA. You're so young. Did it ever occur to you that maybe she loves him? ANA. I'm sure she does. (1.2.34)

De esta conversación podemos extraer varias posiciones. En primera instancia, la posición de la mujer agredida.
La pregunta que hace no es cómo se puede librar de su esposo abusivo, o cómo denunciarlo. Lo que pregunta es ¿cómo hablarle cuando está enojado? Esto es un indicador de una posición de total sumisión ante una realidad que la incomoda pero de la que reconoce que no puede escapar. Por otro lado, la afirmación de Carmen de que ella tiene "suerte" porque su esposo no la golpea, indica una posición similar, en tanto que si se diera el caso, ella tendría que soportarlo. Pancha añade el elemento del amor a la fórmula como un impedimento para separarse de un cónyuge agresor; no obstante, ella no toma en consideración la reciprocidad de dicho amor, en tanto que un ser que agrede a otro no puede amarlo realmente. Ana es la única que reconoce la necesidad de reapropiarse del propio cuerpo y de no aceptar una dominación violenta; no obstante, por primera vez toma en cuenta factores como la dependencia económica de muchas mujeres para con sus esposos, la religión y los hijos como elementos que detienen a muchas mujeres de dejar a un agresor, elementos que para una mujer inmigrante en un país hostil se tornan aún más determinantes.

Por otro lado, la imperante necesidad de ajustarse al ideal de belleza que la sociedad le impone a los cuerpos de las mujeres, lleva a nuestros personajes a pensar constantemente en su "problema" de sobrepeso. En diferentes ocasiones, Carmen le insiste a Ana que tiene que bajar de peso, alegando que lo hace "por su propio bien" (1.1.910). Esta afirmación denota las consecuencias de no adaptarse a los patrones establecidos: si lo hace le irá bien, de lo contrario sufrirá. Más adelante, Carmen revela cómo la necesidad de la 
mujer de verse bella se da en función del hombre, en tanto es el medio por el cual se puede alcanzar la meta de conseguir a un hombre con el cual casarse.

CARMEN. You know, Ana, you're not bad looking. If you lost 20 pounds you would be very beautiful.[... ]

Why don't you lose weight? Last time you lost weight you were so thin and beautifuller.

ANA. I like myself. Why should I? [...] CARMEN. It's because she's young. At this age young girls should try to make themselves as attractive as possible.

ANA. Why? Why not always? You're overweight too.

CARMEN. But I'm already married. ANA. Is that it? Make yourself attractive so that I can catch a man? (2.3.59)

Para Carmen, la búsqueda de la belleza física no tiene nada que ver con una satisfacción personal, con sentirse bella para ella misma, sino como medio para un fin eminentemente patriarcal. Ana y Estela se rehúsan a participar de la objetivación de sus cuerpos. Ana comenta, "Amá, I do want to lose weight. But part of me doesn't because my weight says to everyone, 'Fuck you!' ... It says, 'How dare you try to define me and tell me what I have to be and look like!' So I keep it on. I don't want to be a sex object" (2.3.59); Estela, por su parte, afirma que "I want to be taken seriously, to be considered a person..." (2.3. 59) y no como un objeto sexual. Cuando Estela planea su cita con "el Tormento" surge la discusión,

CARMEN. So what are you going to wear? Don't go dressing up like a scarecrow now.
ESTELA. I don't dress like that.

CARMEN. That's why you scare them away.

ESTELA. Como es, Amá. He likes me for me. Didn't you hear? He said I'm intelligent. He doesn't care how I dress. (1. 2.32)

Mientras Carmen se preocupa por la forma en que su hija se viste, en tanto aleja a los posibles candidatos al matrimonio, Estela se preocupa por ser aceptada como lo que es, una mujer inteligente. Si bien "el Tormento" no resultó ser el tipo de hombre que Estela esperaba, ella se mantiene firme en su posición. En un punto de la obra, Ana logra convencer a las mujeres de mostrar su cuerpo con orgullo, cuando una a una se desprenden de sus ropas para comparar sus "defectos",

CARMEN. (fanning her breasts). Pero que bien se siente. It feels so good to be rid of these clothes and let it all hang out.

ANA. Pues sí. Nobody is watching us. Who cares how we look.

ESTELLA. So this is how we look without clothes?

CARMEN. Just as fat and beautiful... (2.3.61)

Este ejercicio les ayuda a darse cuenta de que todas son hermosas en su "imperfección"; no obstante, algunos personajes aceptan la imposición de patrones de belleza a sus cuerpos. Rosalí es quizá la más obsesionada con su apariencia física, e incluso cuestiona la posición de Ana y Estela, "What's wrong with being a sex object? What's wrong with wanting to be thin and sexy?" (2.3.59). Alentada hasta cierto punto por sus compañeras y por 
su propia ocupación -los vestidos que ellas confeccionan son diseñados para mujeres delgadas, por lo que ninguna de ellas puede usarlos-, Rosalí está dispuesta a convertirse en una mujer delgada, en ese objeto sexual que es su meta, incluso a costa de su propia salud. Comentan,

[...] (ESTELA finishes ironing the dress. She shakes it a bit then puts it on the mannequin. All the WOMEN stare at the dress.)

ROSALI. Que bonito. How I would like to wear a dress like that.

PANCHA. But first you have to turn into a stick to wear something like that. ROSALI. Yeah, but they're worth it. (1.1.22)

La afirmación de que "they're worth it" (valen la pena) demuestra la disposición de Rosalí a asumir cualquier riesgo con tal de lograr su objetivo. Se somete a una peligrosa dieta a base de agua y píldoras para adelgazar que le causan un desmayo. Rosalí explica,

ROSALI. It wasn't just the heat... I hadn't eaten and that's why I fainted. I didn't want you to think it was your fault.

PANCHA. But why do you need to lose weight? 'Tas flaca. [...]

ANA. Rosalí, you can't see yourself the way we see you and that's why you think you're fat. [...]

ROSALI. I'm not hungry because I've been living on diet pills. [...]

When I fainted I saw my body lying there, I thought I was going to die. I couldn't feel my body. (2.1.54)

Rosalí lleva su cuerpo al límite con tal de sentirse hermosa y deseada por primera vez en su vida, ya que la carga por la belleza, representada por la mujer delgada, siempre la ha atormentado hasta el punto de evitar tener relaciones sexuales: “... I've felt fat ever since I can remember and didn't want anybody to touch me until I got thin" (2.3.59). Incluso las otras mujeres comentan sobre la conexión entre el peso y el sexo. Mientras miran un libro pornográfico llamado "Two Hundred Sexual Positions Illustrated", nos dicen,

CARMEN. Ahh. She's so big. No le da vergüenza.

ROSALI. I didn't know they had large women in porno books.

PANCHA. I guess some men enjoy watching big women. [...]

ESTELA. People this fat shouldn't be having sex! Ichhh! (1.2.25)

Estos comentarios reflejan la relación entre el ideal de belleza y la posibilidad de disfrutar del sexo, actividad que ya de por sí está regulada por patrones patriarcales represivos.

La paradoja en toda esta construcción del cuerpo de la mujer radica en que por un lado se insiste en mantener a la mujer como un ser "moralmente correcto" en lo sexual, un ser cuya sexualidad se limita a la concepción de los hijos y, por otro lado, se utiliza y se convierte a la mujer en objeto sexualmente explotado tanto en el acto mismo del coito como en el imaginario colectivo -en los medios de comunicación y en las constantes imágenes de cuerpos femeninos que nos bombardean a diario- mas imposibilitado a disfrutar de la relación. Millet comenta al respecto,

One also observes the paradoxical situation that while patriarchy tends to 
convert woman to a sexual object, she has not been encouraged to enjoy the sexuality which is agreed to be her fate. Instead, she is made to suffer for and be ashamed of her sexuality, while in general not permitted to rise above the level of a nearly exclusively sexual existence. For the great mass of women throughout history have been confined to the cultural level of animal life in providing the male with sexual outlet and exercising the animal functions of reproduction and care of the young. (119)

La sexualidad de muchas mujeres se limita a complacer al hombre, mientras que se les ha obligado a mantener "su pureza". "Woman" nos dice Millet, "is still denied sexual freedom and the biological control over her body through the cult of virginity, the double standard, the proscription against abortion, and in many places because contraception is physically or psychically unavailable to her" (54). Así, la dominación masculina sobre el cuerpo de la mujer la pone en un conflicto en el que se enfrentan sus propios deseos a los deseos impuestos por una sociedad patriarcal, su propia percepción de ella misma a la percepción que la sociedad tiene de ella, su deseo de controlar su propio cuerpo a la imposición de habitar un cuerpo que no le pertenece.

Ana, a pesar de ser la más joven y la que alega no haber tenido relaciones sexuales aún, parece ser la que tiene más conocimiento acerca del sexo, incluso más que su madre, o al menos es la que se atreve a reconocerse como un ser sexual. Ella confronta a las otras mujeres a que se expresen y externen sus dudas, "Go ahead. Ask me anything you always wanted to know about sex but were afraid to ask. I'll tell you (All the WOMEN are tempted.) (1.2.27). Por el contrario, los demás personajes ven el conocimiento sobre sexo como algo contraproducente, "Your husband's not going to like you knowing so much", le dice Carmen a Ana, y Pancha la secunda diciendo "A girl shouldn't know so much" (1.2.27). El conocimiento que Carmen tiene sobre sexo viene totalmente de su experiencia y de lo que su esposo le enseña. Ella cuenta su primera experiencia sexual,

CARMEN. ... Okey, pues after riding on his bike for so long, I had to pee so bad! So we stopped in the mountains somewhere. I ran behind a tree, squatted, and just peed. That night, after we got settled, I didn't know what was going to happen. After we did it, I started itching and scratching down there 'til my cuchupeta got so red. I thought something was wrong, but I asked him and he said it was suppose to hurt and bleed. Then I found out it wasn't him. I had peed on poison ivy. And how it hurt! (1.2.28, la cursiva es mía)

Esta graciosa historia pone de manifiesto la total ignorancia con la que muchas mujeres llegan al momento del acto sexual. Ella desconocía las posibles reacciones que su cuerpo podía tener. Es su marido, el "dueño" de su cuerpo, el que, sin ser mujer, le enseña lo que se supone tiene que pasar desde su perspectiva. Ahora bien, a pesar de que Ana tiene una visión de la sexualidad mucho más libre que sus compañeras, de igual forma esa expresión de libertad sexual se limita a espacios de mujeres, en tanto que la presencia de la autoridad masculina la disuadiría de ese tipo de comentarios. Carmen 
la reprende invocando la figura paterna, “iHíjole! If your Apá were to hear you... ¡Híjole!", a lo que Ana responde "I wouldn't be talking like this in front of my father" (1.2.27). Esto refleja el hecho de que Ana no ha logrado cortar por completo con la dominación que ejerce su padre sobre su cuerpo, a pesar de encontrarse en una posición mucho más libre que las otras mujeres.

Para Carmen, ser mujer, convertirse en mujer es más un paso de la niñez a la maternidad que se alcanza al perder la virginidad, "In my day, a girl became a woman when she lost her virginity" (1.2.27). La llamada "pérdida de inocencia" es solo compensada en tanto la mujer se convierta en madre. La maternidad regulada representa el único fin por el cual se le permite a la mujer participar del acto sexual sin tener que cargar con todo el peso de la moral. Cixous explica que "... desde siempre, se supone que, estando encinta, la mujer no sólo dobla su valor mercantil, sino sobre todo, se valoriza como mujer ante sí misma, y cobra cuerpo y sexo indudables" (52). Es en este momento en que la mujer comienza a tener valor como tal ante los ojos del patriarcado, y lo será hasta el momento en que le sea físicamente imposible, "Ella se levanta", agrega Cixous, "- final del sueño -, la continuación es sociocultural, él le hace muchos hijos, ella se pasa la juventud pariendo; de cama en cama, hasta la edad en que deja de ser mujer" (18). Esta misma forma de pensar la comparten Carmen y Pancha, quienes ven la maternidad como una responsabilidad de toda mujer y como el fin que las determina como tales. Ante la posibilidad de estar embarazada de nuevo, Carmen externa su preocupación y su experiencia,
ANA. Amá, do you really want to have it? PANCHA. Doña Carmen, give it to me if you don't want it.

CARMEN. I can't just get rid of it, either way... But I don't want to have it. PANCHA.Butyou'relucky,DoñaCarmen. CARMEN. No. it seems all I do is have children. One after the another. i'm tired of this! I can't have this baby. I'll die. Last time I was pregnant the doctor said I almost didn't make it.

ANA. Amá, I didn't know that happened. CARMEN. Every time your Apá touches me, the next day I'm pregnant. When he would leave me in Mexico to go to el norte, he would leave me pregnant so no man would look at me and desire me. I was very beautiful.

ANA. You still are, Amá.

CARMEN. I was always scared of him. And I let myself get fat after you were born hoping he would be disgusted by me and not touch me anymore.

ANA. Why didn't you just say "No"?

CARMEN. Because, M'ija, I was never taught how to say no. (1.3.57)

Por un lado, Carmen revela su imposibilidad de negarse a su rol de madre, en tanto su marido tiene total dominio sobre su cuerpo, y se ve obligada a recurrir a técnicas tales como descuidar su apariencia física con tal de disuadir indirectamente a su marido de embarazarla nuevamente. Por otro lado, vemos la posición de Pancha, quien añora tener la posibilidad de quedar embarazada y convertirse en una "verdadera" mujer. A solas comenta, "Que bonito viento. Wind, that's what I am. (Touching her stomach.) Empty, like and old rag... (Praying.) Diosito, why don't you make me a real woman? If I can't have children, why did you make me a woman?" (2.1.52). Para ella, el rol de la 
mujer es ser madre, si no, su existencia no tiene sentido, mucho menos el sexo. Incluso Carmen, quien no desea tener más hijos, al darse cuenta de que los síntomas que tiene no son de embarazo sino de menopausia, expresa su malestar "when you reach menopause it's over. You're no longer a woman. Se te seca allí abajo" (2. 4.65).

Finalmente Ana exhorta a las otras mujeres a liberarse de sus ataduras y a decir no,

But we can't allow ourselves to be abused anymore. We have to assert ourselves. We have to realize that we have rights! We have the right to control our bodies. The right to exercise our sexuality. And the right to take control of our destiny. But it all begins when we start saying... (ANA quickly climbs on top of a sewing machine to continue preaching) ... ¡Ya basta! No more! We should learn how to say no! Come on, Amá, say it! Say it! (1.3.34)

No obstante, tal y como lo expuso Carmen anteriormente, decir "no", como un acto realmente asertivo y de resistencia, es algo que debe aprenderse con el tiempo, no es algo que ellas puedan decir realmente de la noche a la mañana en tanto que los hábitos de la dominación están arraigados en la cultura, en la vida diaria, en la realidad de estas mujeres.

En Real Women Have Curves, Josefina López explora los diferentes puntos de vista de mujeres que, a pesar de compartir ciertas características que las unifican, también viven realidades que las separan totalmente en su percepción del "ser mujer". La perspectiva de Ana, que se asemeja más a la perspectiva de una feminista del
Primer Mundo en su forma de pensar, descubre que su conocimiento, su libertad, su forma de ver el mundo, no necesariamente pueden cambiar la forma en que mujeres de contextos distintos piensan y viven sus vidas, y de cómo sus propias condiciones de existencia determinan en cierta medida quién es y hacia dónde va. Ella descubre que su feminismo "gringo" se torna etnocéntrico, al subestimar aspectos tales como la condición económica, la nacionalidad, la edad, la cultura, como elementos claves en la construcción de las identidades de cada mujer. Incluso la misma Ana se muestra aún atada a ciertas condiciones de dominación que la sociedad en la que se desarrolla le impone, en tanto el ejercicio de su voz lo hace libremente en ausencia de una figura masculina, mientras que su situación económica la limita en su desarrollo profesional y académico. Ana aprende a no juzgar a sus compañeras por querer ser madres, por negarse a abortar, por someterse a dietas asesinas, por creer en la virginidad, en el matrimonio, ya que son todos estos elementos aún parte del bagaje cultural del que provienen; no obstante, las demás mujeres aprenden también de Ana que existe un mundo distinto en el que ellas pueden tomar el control de sus cuerpos, en que la mujer se determina por lo que es - un ser humano-y no por su apariencia o su capacidad de engendrar bebés. Esta obra es una muestra de lo que el feminismo multicultural, poscolonial debe enfrentar para no caer en un discurso totalizador y esencialista del ser mujer. Un feminismo que analice las variables culturales, económicas, discursivas y demás, que intervienen en la construcción de los sujetos, tanto hombres como mujeres, 
para así poder ofrecer oportunidades realistas de cambio acordes con las particularidades de cada grupo.

\section{Notas}

1 Incluso en el contexto occidental, las mujeres son atravesadas por particularidades de clase social, educación, etc., que las definen, de forma distinta, dentro del marco del Primer Mundo.

2 Pensamos el Tercer Mundo como colonial o poscolonial en su basta mayoría, si tomamos en cuenta no solo la dependencia por la toma de un territorio por parte de un imperio, sino también procesos de dependencia incluso económica.

\section{Bibliografía}

Althusser, Louis. "Ideology and Ideological State Apparatuses." Lenin and Philosophy and other Essays. New York: Monthly Review Press, 1971. Impreso.

Belausteguigoitia, Marisa. "Descaradas y deslenguadas: el cuerpo y la lengua india en los umbrales de la nación." Debate Feminista. Racismo y Mestizaje 12.24 (2001): 230254. Archivo PDF.

Bourdieu,Pierre. "Unaimagenaumentada." La dominación masculina. Barcelona: Anagrama, 2000. Impreso.

Butler, Judith. "Sujetos de sexo/género/deseo." El género en disputa. El feminismo y la subversión de la identidad. México D.F.: UNAM, 1999. Impreso.

Cixous, Hélène. "La joven nacida." Parte II. La risa de la medusa. Barcelona: Anthropos, 2001. Impreso.

Femenías, María Luisa. "El feminismo postcolonial y sus límites." Teoría feminista: de la Ilustración a la globalización. Ed. Celia Amorós y Ana de Miguel. Madrid: Minerva, 2005. Impreso.

Hernández Castillo, Aída. "Entre el etnocentrismo feminista y el esencialismo étnico. Las mujeres indígenas y sus demandas de género." Debate Feminista: Racismo y Mestizaje 12.24 (2001): 206-229. Archivo PDF.

López, Josefina. Real Women Have Curves. Illinois: Dramatic Publishing, 1990. Impreso.

Millet, Kate. Sexual Politics. Urbana and Chicago: University of Illinois Press, 2000. Impreso.

Mohanty, Chandra Talpade. "De vuelta a 'Bajo los ojos de Occidente': la solidaridad feminista a través de las luchas anticapitalistas." Descolonizando el Feminismo: Teoría y práctica desde los márgenes. Ed. Liliana Suárez y Aída Hernández. Madird: Cátedra, 2008. Impreso.

Young, Robert J.C. "Postcolonial Feminism" Post-Colonialism: A Very Short Introduction. Oxford: OUP, 2003. Impreso. 
